\title{
Demographics of the Profession
}

\author{
ERIN MCGRATH | AMERICAN POLITICAL SCIENCE ASSOCIATION
}

he American Political Science Association serves a diverse membership in the United States and around the globe. From 2015 onwards, APSA required members to opt in or opt out of providing demographic information. We compared APSA's administrative membership data for the period 2017-2019 (pre-COVID-19 pandemic) to data from the NSF's Survey of Earned Doctorates of PhDs awarded in political science or government for 2019. Our findings are illustrated below.

Figure 1: NSF Survey of Earned Doctorates: PhDs Awarded in Political Science or Government in 2019

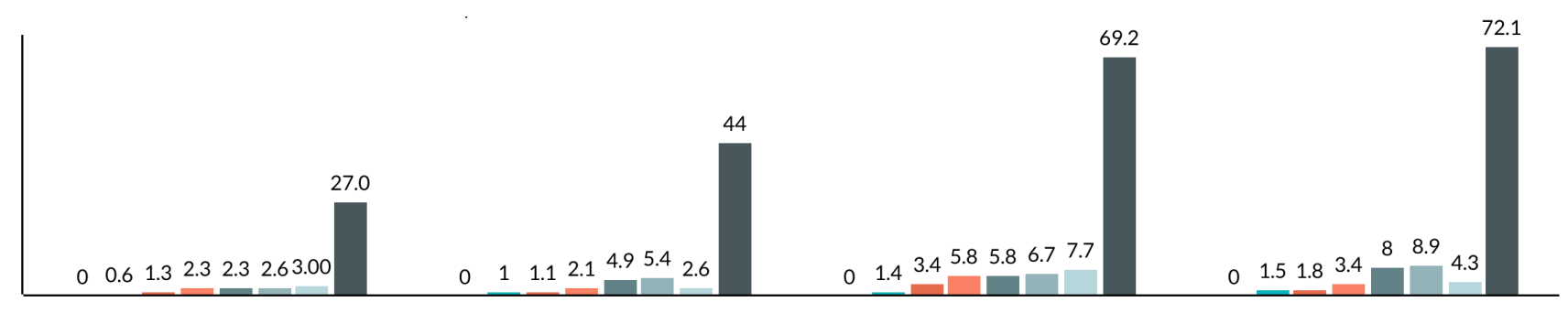

female $x$ race/eth as $\%$ of total

male $x$ race/eth as \% of total

female $x$ race eth as \% of female

male $x$ race eth as $\%$ of male

Native American or Alaska Native Middle Eastern or Arab American

Other

Black, Afro-Caribbean, or African American
Latino or Hispanic American

East Asian or Asian American

Prefer not to disclose

Non-Hispanic White or Euro-American
All figures presented are derived from administrative data on APSA membership. *Non-binary genders accounted for less than $1 \%$ when divided into race/ethnic categories.

Figure 2: Pre-COVID Proportions of Groups of Color Among Binary Genders, 2017-2019*
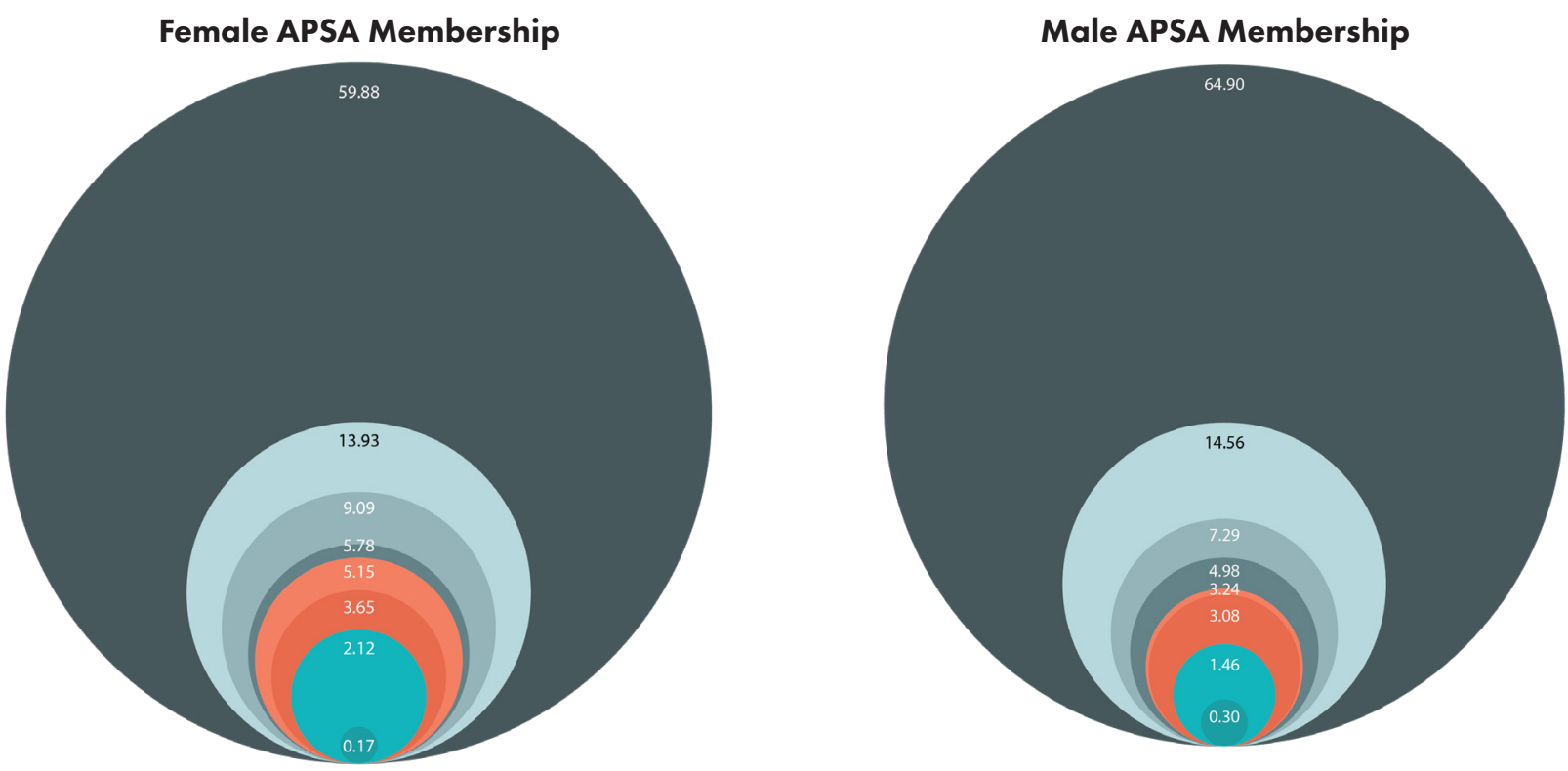
Figure 3: Female Race/Ethnicity as \% of Total Membership

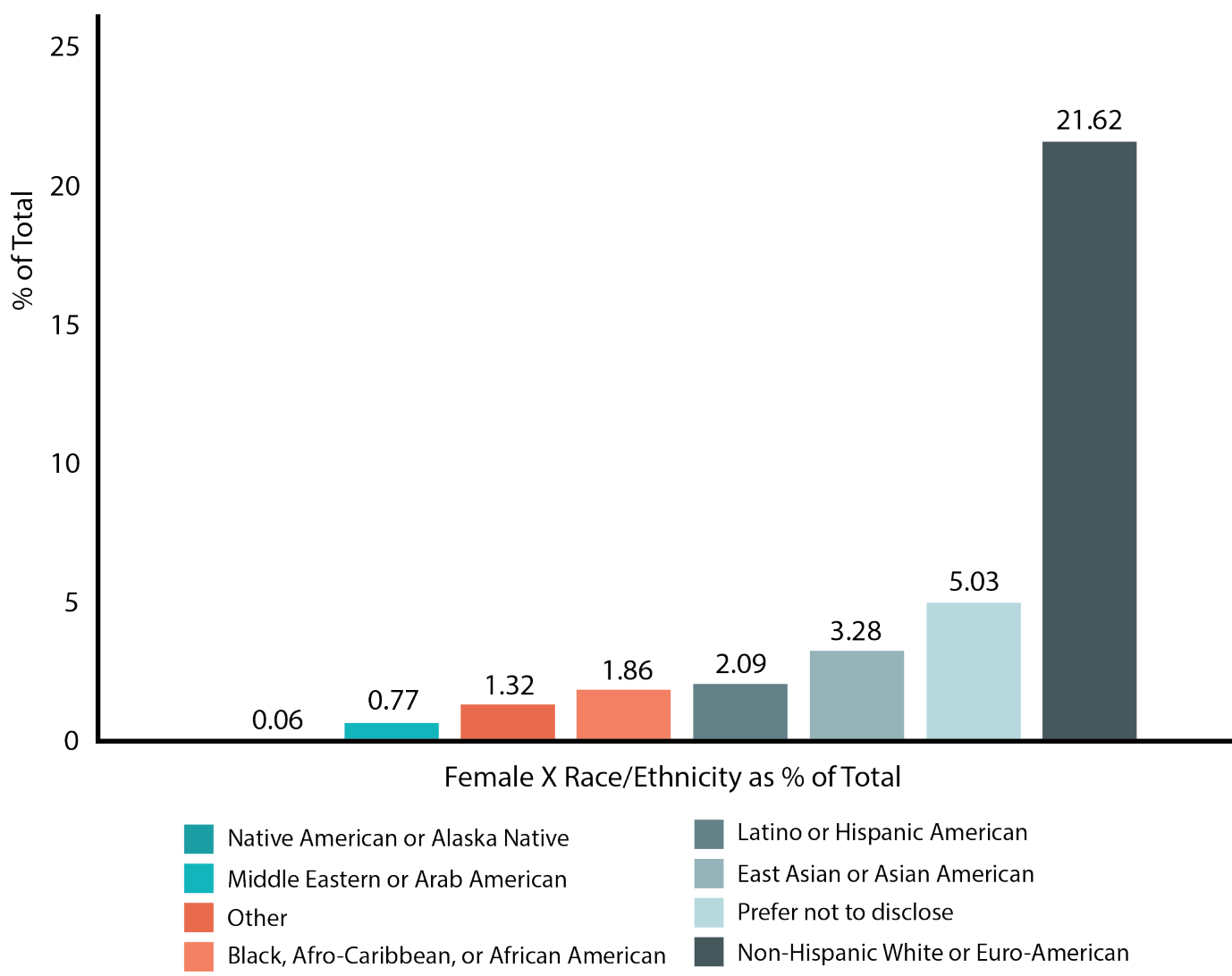

Figure 4: Male Race/Ethnicity as \% of Total Membership

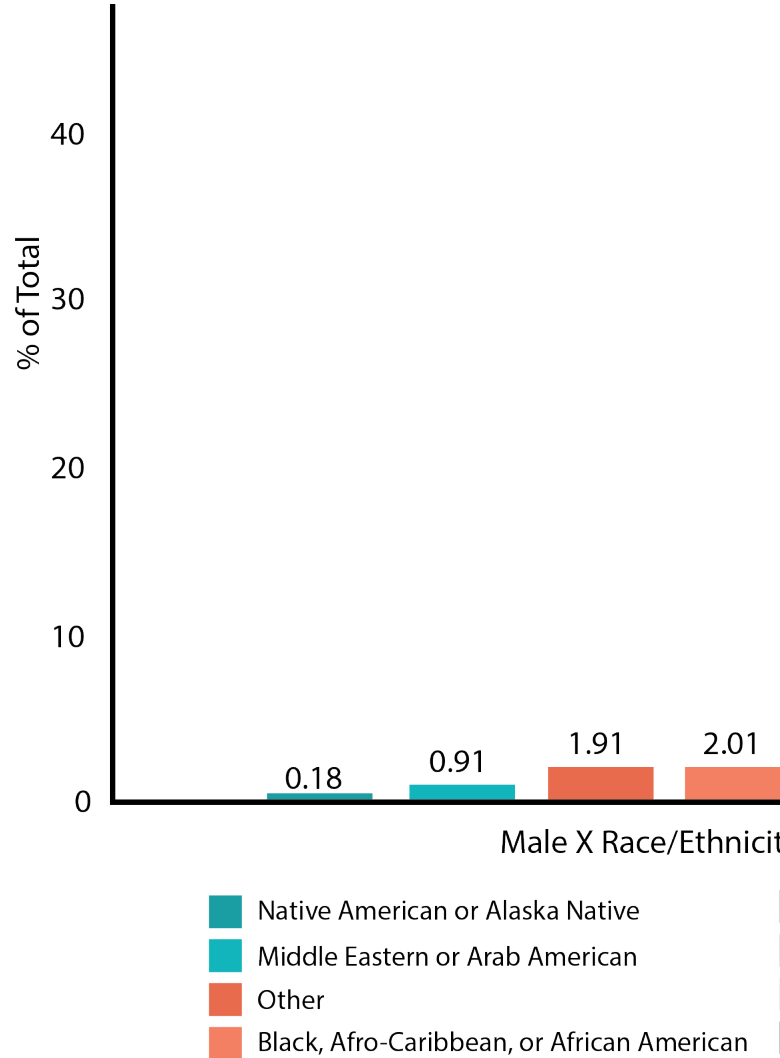

Latino or Hispanic American

East Asian or Asian American

Prefer not to disclose

Non-Hispanic White or Euro-American 